\title{
Provider alerts and reminders to improve tuberculosis care among people living with HIV in Kenya: TB Tech formative report
}

\author{
Caricia Catalani \\ Eric P. Green \\ Lameck Diero \\ Jane Carter \\ Martin Were
}

See next page for additional authors

Follow this and additional works at: https://knowledgecommons.popcouncil.org/departments_sbsr-hiv

Part of the Demography, Population, and Ecology Commons, Family, Life Course, and Society Commons, and the International Public Health Commons How does access to this work benefit you? Let us know!

\section{Recommended Citation}

Catalani, Caricia, Eric P. Green, Lameck Diero, Jane Carter, Martin Were, Dennis Israelski, Adrian Gardner, Charity Ndwiga, and Paul Biondich. 2014. "Provider alerts and reminders to improve tuberculosis care among people living with HIV in Kenya: TB Tech formative report." Washington, DC: Population Council. 


\section{Authors}

Caricia Catalani, Eric P. Green, Lameck Diero, Jane Carter, Martin Were, Dennis Israelski, Adrian Gardner, Charity Ndwiga, and Paul Biondich 
瑟 PEPFAR

\section{PROVIDER ALERTS AND REMINDERS TO} IMPROVE TUBERCULOSIS CARE AMONG PEOPLE LIVING WITH HIV IN KENYA: TB TECH FORMATIVE REPORT

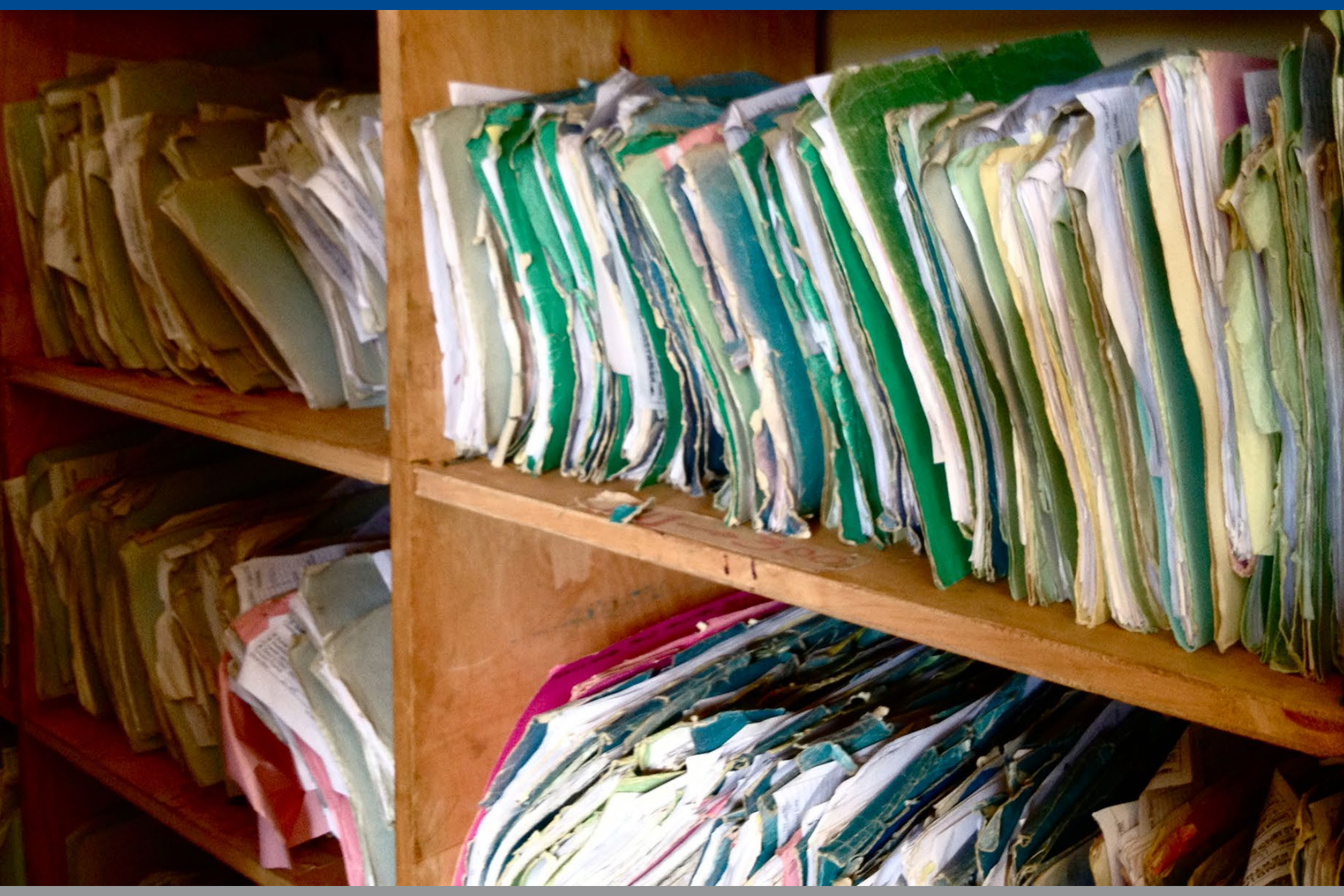





\section{PROVIDER ALERTS AND REMINDERS TO IMPROVE TUBERCULOSIS CARE AMONG PEOPLE LIVING WITH HIV IN KENYA: TB TECH FORMATIVE REPORT}

CARICIA CATALANI, ERIC P. GREEN, LAMECK DIERO, JANE CARTER, MARTINWERE, DENNIS ISRAELSKI,ADRIAN GARDNER, CHARITY NDWIGA, AND PAUL BIONDICH

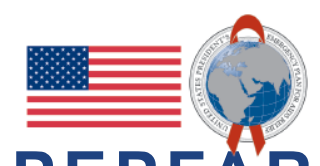
PEPFAR 


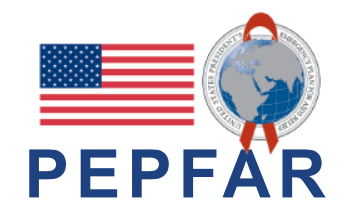

This report was made possible through support provided by the President's Emergency Plan for AIDS Relief and the U.S. Agency for International Development (USAID) via HIVCore, a Task Order funded by USAID under the Project SEARCH indefinite quantity contract (Contract No. AID-OAA-TO-11-00060).

\section{HIVCore improves the efficiency, effectiveness, scale, and quality of HIV treatment, care, and support, and prevention of mother-to-child transmission (PMTCT) programs. The Task Order is led by the Population Council in partnership with the Futures Group, the Elizabeth Glaser Pediatric AIDS Foundation, and the University of Washington.}

Published in May 2014. (C2014. The Population Council Inc.

Cover photo credit: Caricia Catalani, InSTEDD

Suggested citation: Catalani, Caricia, Eric P. Green, Lameck Diero, Jane Carter, Martin Were, Dennis Israelski, Adrian Gardner, Charity Ndwiga, and Paul Biondich. 2014. "Provider alerts and reminders to improve tuberculosis care among people living with HIV in Kenya: TB Tech formative report,” HIVCore Formative Report. Washington, DC: USAID | Project Search: HIVCore. 


\section{TABLE OF CONTENTS}

\section{ACRONYMS}

INTRODUCTION

FORMATIVE RESEARCH 3

KEY FINDINGS $\quad 4$

Preparedness to screen for TB and provide IPT 4

Preparedness to capture and report critical indicators of system performance 5

Usability of TB reminders 6

CONCLUSIONS AND RECOMMENDATIONS 8 


\section{ACRONYMS}

AFB

AMPATH

AMRS

CHW

CXR

HIV

IPT

$\mathrm{MOH}$

PLHIV

TB

WHO
Acid-Fast Bacilli

Academic Model Providing Access to Healthcare

AMPATH medical record system

Community health worker

Chest X-ray

Human immunodeficiency virus

Isoniazid preventive therapy

Ministry of Health

People living with HIV

Tuberculosis

World Health Organization 


\section{INTRODUCTION}

People living with HIV (PLHIV) have a 20-fold higher risk of dying from tuberculosis (TB) than the general population. Reducing TB morbidity and mortality among PLHIV requires identifying those with active TB and treating them, as well as preventing new TB infections among those who are not infected. The World Health Organization (WHO) recommends screening all HIV-infected patients for symptoms of active TB infection, testing those who show symptoms, treating those with positive TB tests, and providing isoniazid preventive therapy (IPT) for those who are either asymptomatic or whose TB test results are negative.

WHO classifies Kenya among the "high burden" countries for TB and notes high rates of HIV-TB coinfection. ${ }^{\text {T The }} 2012$ Global Tuberculosis Report also singles out Kenya and Rwanda as "top performers" in testing TB patients for HIV and provision of ART. Equally important is screening and testing HIVinfected patients for TB, which is the focus of this report.

The Academic Model Providing Access to Healthcare (AMPATH) consortium—a partnership between Indiana University in the United States, Moi University School of Medicine in Kenya, and several other medical schools - works in partnership with the Kenyan Ministry of Health (MOH) to manage HIV care across western Kenya. Its catchment area includes over 40 medical sites providing HIV care with approximately 40,000 HIV patient visits per month. Tuberculosis risks to these patients are substantial: 23 percent of adults in the region have latent $\mathrm{TB}$ infection, and 48 percent of patients with active TB have HIV co-infection.

TB testing and diagnosis are decentralized in the AMPATH system, meaning that testing can be initiated at all levels, from the community to service facilities. Sputum is analyzed by smear microscopy, and several higher-level labs use GeneXpert to verify select sample results. Although WHO standards do not specifically require it, AMPATH also uses chest X-ray for TB diagnosis. AMPATH has gone to great lengths to ensure that X-ray facilities are available as far and wide as possible, including mobile X-ray vehicles.

AMPATH has clear standards of care to address TB among HIV-infected patients. Implementing these standards of care requires clinicians to screen HIV-infected patients for TB symptoms during routine clinical encounters.

Ensuring understanding and compliance with these standards among providers across a complex and low-resource health system has been challenging. In a study of IPT initiation conducted between 2004 and 2008, Carter et al. (2012) found that 38.1 percent of eligible patients initiated IPT ${ }^{2}$. Given the importance of IPT at AMPATH and among PLHIV worldwide, this intervention is critical. It was

\footnotetext{
${ }^{1}$ World Health Organization. “Global tuberculosis report 2012.” http://apps.who.int/iris/bitstream/10665/75938/1/
} 9789241564502_eng.pdf

${ }^{2}$ Carter, E. et al. 2012. "The use of isoniazid preventive therapy in an HIV care and treatment program in Western Kenya." 
hypothesized that using information from patient electronic medical records to give medical providers tailored reminders could improve three outcomes: (a) screening for TB; (b) initiation of IPT among HIV-positive adults who do not have active TB; and (c) completion of IPT. To test this hypothesis, the “TB Tech" study, under the U.S. Agency for International Development's (USAID's) HIVCore project led by the Population Council, was initiated by researchers from Kijani Consulting, InSTEDD, Regenstrief Institute, Moi University in partnership with other AMPATH consortium members also.

In order for this system to work, there needs to be (a) staff who are equipped to screen, test, and prescribe IPT and (b) an information system that uses patient data to determine the timing and content of the reminders, and a strategy for testing, monitoring, and evaluating the usefulness and effectiveness of the reminders. To assess these two dimensions, the study team conducted formative research to address three critical areas:

- Preparedness of AMPATH facilities and providers to screen for TB symptoms and provide IPT for symptom-negative HIV-infected patients.

- Preparedness of the AMPATH medical record system $\left(\right.$ AMRS $\left.^{3}\right)$ to capture and report critical indicators of IPT/TB service performance.

- Preparedness of the AMPATH AMRS as well as other data sources to capture and report critical indicators of reminder system performance.

\footnotetext{
${ }^{3} \mathrm{AMRS}$ is built on OpenMRS, an open source software platform and reference application. To date, the AMRS has collected more than 100 million discrete clinical observations from 2.8 million AMPATH visits made by 300,000 enrolled patients. Stored data include test results; patient descriptors such as symptoms, vital signs, and physical exam findings; and diagnoses and treatments from clinical encounters.
} 


\section{FORMATIVE RESEARCH}

Formative research began in January 2013 and was planned to continue through August. It included direct observation, key informant interviews, and document review. A diverse range of sites were identified through purposeful selection across the following key characteristics: rural/urban, average monthly patient volume in 2012, total number of active providers in 2012, and AMPATH system clinic level (1-6). Table 1 summarizes the data collection methods. Data were collected by the study investigators.

Table I Formative research methodology

\begin{tabular}{|lll|}
\hline Strategy & \multicolumn{1}{c|}{ Number } & \multicolumn{1}{c|}{ Details } \\
Site observations & 9 sites & Semi-structured observation guide. \\
Key informant discussions & $\begin{array}{l}24 \text { key } \\
\text { informants }\end{array}$ & $\begin{array}{l}\text { Unstructured interview guide. Medical superintendents, } \\
\text { clinicians, Ministry of Health officials, laboratory managers, } \\
\text { pharmacy managers, medical directors, TB care providers, } \\
\text { AMPATH administrators and program managers, data quality } \\
\text { workers, and community health workers (CHWs). }\end{array}$ \\
Usability assessment & 6 clinicians & $\begin{array}{l}\text { A mixed-methods usability survey and in-depth interview were } \\
\text { used to assess clinician's perceptions of the understandability, } \\
\text { importance, helpfulness, practicality/feasibility, and accuracy of } \\
\text { prototype TB reminders introduced throughout a day of regular } \\
\text { clinical duties. }\end{array}$ \\
Program document review & N/A & Inventory of all forms found in AMPATH facilities.
\end{tabular}




\section{KEY FINDINGS}

\section{PREPAREDNESSTO SCREEN FORTB AND PROVIDE IPT}

The AMPATH system provides multiple opportunities to integrate TB screening into routine HIV care, including clinical forms and community outreach. While there are several successes with the current AMPATH algorithm, the system still has some challenges which need to be addressed for it to function optimally.

The Initial Encounter Form and Return Encounter Form include a number of TB-related questions, including symptoms of active TB. However, a number of key informants, including clinical providers, suspected that clinicians may be checking for symptoms without being aware that they are screening for TB. In addition, AMPATH engages cough monitors — specially trained community health workers- to provide targeted TB education and TB screening at the community level. A screening tool was designed for cough monitors.

Due to the complexity of synchronizing sputum analyses (including microscopy and Xpert) with chest X-ray (CXR), TB diagnosis can be slowed or halted if there are breakdowns in X-ray functionality or delays in returning test results from the lab. For example, if X-ray facilities are down when a provider orders an X-ray, this could delay TB diagnosis for months. Further review of the AMRS is underway to quantify this delay. AMPATH is currently exploring the use of the Xpert molecular testing in cases of AFB negative sputum results among symptom-positive patients.

Site visits discovered that major stock-outs of isoniazid had occurred within the last year. Stocks have been replenished and AMPATH's central pharmacy is now committed to ensuring 3-month stock for all sites. Nevertheless, quick observations of pharmacies suggested that few clinics had adequate stock on hand for widespread IPT. Key informants tended to agree that providers regularly initiated IPT among their HIVpositive/TB-positive patients before the stock-outs. Since then, providers have become wary of starting patients on IPT without adequate supplies to complete a full six-to-nine month course of prophylaxis. Several mentioned that while they still prioritize initiating prophylaxis among the children of TB-positive patients, there is no longer any systematic effort to put their adult patients on IPT. There was no mention of prioritizing other high-risk groups, such as HIV-positive household members or partners of TBpositive patients.

Observers also noted that there might be additional attitudinal barriers to IPT initiation among providers. For example, some providers may not believe that it is effective, that TB is a high priority, or that TB prophylaxis drugs and HIV treatment drugs can be simultaneously managed and tolerated by their patients.

Key stakeholders report that adherence to TB prophylaxis is high among AMPATH patients. However, it is difficult to confirm adherence with existing patient information short of conducting patient interviews, which were beyond the scope of this formative study. 


\section{PREPAREDNESS TO CAPTURE AND REPORT CRITICAL INDICATORS OF SYSTEM PERFORMANCE}

The field visits discovered a number of data forms that might feasibly be used to create provider reminders and to track TB screening, testing, and diagnosis, and IPT initiation and completion. At the same time, the study team observed that IPT records are not integrated into AMRS or any other computerized information system. Rather, initiation and adherence data seem to be inconsistently documented in a paper MOH TB register and IPT register. This information is summarized in the aggregate in quarterly reports.

Table 2 lists the data forms that might be harnessed in the design, implementation, and evaluation of the IPT clinical reminders study.

Table 2 Data forms relevant to TB screening, testing, and IPT

\begin{tabular}{|c|c|c|c|c|}
\hline Description & $\begin{array}{l}\text { Point of } \\
\text { collection }\end{array}$ & $\begin{array}{l}\text { Person } \\
\text { collecting }\end{array}$ & $\begin{array}{l}\text { Computer } \\
\text { entered }\end{array}$ & $\begin{array}{l}\text { Integrated } \\
\text { with AMRS data }\end{array}$ \\
\hline $\begin{array}{l}\text { Cough monitor screening tool } \\
\text { (AMPATH) }\end{array}$ & $\begin{array}{l}\text { Community/ } \\
\text { Household }\end{array}$ & $\begin{array}{l}\text { CHW, Cough } \\
\text { monitor }\end{array}$ & Unknown & Unknown \\
\hline $\begin{array}{l}\text { Isoniazid Preventative Therapy Facility } \\
\text { Registry }(\mathrm{MOH})\end{array}$ & Facility & $\begin{array}{l}\text { Clinical } \\
\text { provider }\end{array}$ & Unknown & Unknown \\
\hline TB Treatment Registry (MOH) & Facility & $\begin{array}{l}\text { Clinical } \\
\text { provider }\end{array}$ & Unknown & Unknown \\
\hline $\begin{array}{l}\text { Pharmacy central sites/ district store } \\
\text { consumption and request (AMPATH) }\end{array}$ & Facility & $\begin{array}{l}\text { Pharmacy } \\
\text { technician }\end{array}$ & Yes & $\mathrm{N} / \mathrm{A}$ \\
\hline $\begin{array}{l}\text { Pharmacy patient pick-up } \\
\text { registry }\end{array}$ & Facility & $\begin{array}{l}\text { Pharmacy } \\
\text { technician }\end{array}$ & Variable & Unknown \\
\hline Patient Encounter Form (AMPATH) & Facility & $\begin{array}{l}\text { Clinical } \\
\text { provider }\end{array}$ & Yes & Yes \\
\hline $\begin{array}{l}\text { Patient Return Encounter Form } \\
\text { (AMPATH) }\end{array}$ & Facility & $\begin{array}{l}\text { Clinical } \\
\text { provider }\end{array}$ & Yes & Yes \\
\hline $\begin{array}{l}\text { Laboratory Information System } \\
\text { (AMPATH) }\end{array}$ & Facility & $\begin{array}{l}\text { Lab } \\
\text { technician }\end{array}$ & Yes & Yes \\
\hline $\begin{array}{l}\text { GeneXpert Information System } \\
\text { (AMPATH) }\end{array}$ & Facility & $\begin{array}{l}\text { Lab } \\
\text { technician }\end{array}$ & Yes & In process \\
\hline Radiology Registry & Facility & $\begin{array}{l}\text { X-ray } \\
\text { technician }\end{array}$ & Unknown & Unknown \\
\hline
\end{tabular}

Before decisions can be made regarding which data forms should be used, the quality of the data collected must be evaluated along several dimensions, including completeness, consistency, validity, conformity, reliability, and integrity. Due to time constraints, a systematic assessment of data quality was not made across all sites visited. Key informant interviews were conducted with the Data Quality Team at AMPATH headquarters and observations made during the first visit, to Eldoret. Several issues and questions requiring further investigation were revealed.

Completeness: To what extent are TB-related data comprehensively captured by the AMRS? According to the Data Quality Team, all items (fields) on the initial and return HIV encounter forms should also 
have a value recorded in the database. When values are missing or illegible, a member of the team flags the item with a paper note and sends the encounter form back to the clinic to be completed. This process should reduce the amount of missing data in the AMRS, but further review and verification of AMRS data completeness are required.

However, many key TB indicators are not included in the AMRS database, rather they are captured elsewhere. For example, medication adherence is recorded in a separate register and patient-level data are not passed to the AMRS. Monitoring patient adherence to IPT will require data assistants to track down data from multiple forms.

Consistency: To what extent are data internally consistent? AMPATH HIV encounter forms are entered into AMRS; a retrospective review of the database will be conducted before designing the reminder algorithms to determine if any fields permit inconsistencies.

Validity: Do the TB fields on the encounter forms measure what they are intended to measure? The forms require the provider to indicate whether a symptom is either present or absent; in other words, if the provider asks the patient if he/she has had a fever and the patient answers no, the provider should mark "no fever" rather than leaving the fever question blank. A missing field may indicate that the question was either not asked or the answer was not recorded (see completeness, above); it is also important to ascertain if when "no fever" is marked, the provider asked the question and the patient answered or the provider completed an overall clinical assessment of the patient and then later responded to the encounter form questions with the information he/she gleaned.

Conformity: Do the TB fields on the encounter forms permit non-standardized responses? All HIV encounter forms have restricted response options and thus only standard responses are submitted.

Reliability: Are the TB-related data stable over time in the absence of "true" changes in the patients' health status? AMRS data queries should help to verify if a patient diagnosed with active TB at a particular point in time remains classified as having active TB during the next two follow-up encounters.

\section{USABILITY OF TB REMINDERS}

At two pre-selected sites, all clinicians working on the day of the visit (a total of six providers) were asked to rate $\mathrm{TB}$ reminder messages on the four usability criteria listed in Table 3 for the patients they had seen that day. Providers rated all reminders that they received, yielding a total of 17 reminder ratings. Each reminder was scored on a scale from 1 (very bad) to 5 (very good) for the

Table 3 Usability ratings

\begin{tabular}{|lcc|}
\hline Criterion & $\begin{array}{c}\text { Range } \\
(\mathbf{n}=\mid \mathbf{7})\end{array}$ & $\begin{array}{c}\text { Mean } \\
(\mathbf{n}=17)\end{array}$ \\
\hline Understandability & $\mathrm{I}-5$ & 5.0 \\
Importance & $\mathrm{I}-5$ & 4.2 \\
Helpfulness & $\mathrm{I}-5$ & 3.6 \\
\hline Practicality/feasibility & $\mathrm{I}-5$ & 3.4 \\
\hline
\end{tabular}
four criteria, as shown in Table 3.

Interestingly, providers often rated the reminders highly but then indicated that they were incorrect for that particular patient or that they were unable to take any action, as seen in Table 4. 
Table 4 Accuracy and actionability of reminders

\begin{tabular}{|c|c|c|c|}
\hline Criterion & Yes & No & Not sure \\
\hline & $\%$ & $\%$ & $\%$ \\
\hline Correct for this patient today & 47 & 41 & 6 \\
\hline Able to take the next step recommended for TB care today & 29 & 59 & 12 \\
\hline
\end{tabular}

In-depth interviews revealed that clinicians rarely have special education or training around TB care and that there is general confusion about AMPATH's norms for TB care. To be effective, reminders should include specific instructions to the clinician on what actions to take moving forward. For example, one clinician asked during an interview:

So, it says to check for chest X-ray. And what if I find the results? Then what do they want me to do? Am I to wait for the next time they come in to find out what I should have done?

It was also found that the proposed new TB reminders overlapped with other existing reminders. Most notably, there is currently a CXR reminder for all patients with no CXR on file. For approximately half of the reminders received by providers, providers pointed out that the reminder was inaccurate because AMRS was lacking CXR data. Providers found missing CXR data by examining the patient paper record and, more often, by patient self-report. There are various client flow issues affecting the ability of clinicians to accurately document CXR results, as many patients must go on their own to seek CXRs offsite following a clinical encounter.

Finally, providers asked for help not only to identify patients needing IPT but also to provide reminders about appropriate treatment for patients with active TB. Providers made comments like, "so what if a patient does have a cough or other symptoms, the reminders can't end then" and "it would be really helpful to have a reminder about taking a sputum sample, because we often lose a lot of time doing this." As such, several providers explained that they would be more comfortable relying on a TB alert/reminder system that considered both sides of the treatment algorithm-IPT for patients without active TB; and treatment for patients with active TB. Although patients with active TB are sometimes transferred to different providers in different parts of the clinic for infection control, reminders during the early stages of TB diagnosis were important to all providers.

The information presented in this report was collected and assessed with the objective of improving the quality of our reminder system, the quality of AMRS data, and the rigor of our upcoming impact evaluation. As selection of participating individuals and sites was purposeful, and not representative, these findings are not generalizable to AMPATH or other HIV care systems. Much of the information presented in this report was derived from discussion with key informants and clinicians, who may have been unable or unwilling to fully disclose their concerns or critiques of the institution of their employment. More importantly, this information was collected during a limited number of months in 2013 and, as many AMPATH TB experts explained and IPT initiation data confirm, is likely to reflect a particular and challenging time for the distribution of IPT in the history of AMPATH. 


\section{CONCLUSIONS AND RECOMMENDATIONS}

The findings of the formative research demonstrate both strengths and challenges in the preparedness of the AMPATH system to undertake a pilot test of clinical reminders to screen for active TB infection and initiate and complete IPT.

Strengths include the following:

- AMPATH standards of care include screening for TB and providing IPT.

- AMPATH data forms include most of the data needed to generate clinical reminders and track system performance.

- Many of the needed data are already consolidated into electronic patient medical records.

At the same time, the following challenges are noted, which have implications for the pilot test of the clinical reminders:

- Lack of information flow between TB and HIV services. TB treatment providers have limited or no access to the AMRS, and HIV providers have little or no access to their patients' TB treatment status, resulting in an HIV system that does not integrate TB care and a TB system that does not integrate HIV care.

- Isoniazid stock levels are sufficient at AMPATH central pharmacy, but additional demand during the trial might require more stock to be delivered at greater frequency to health facilities.

- Providers do not appear to be prescribing IPT for TB-negative PLHIV; many may be reluctant to prescribe if pharmacy stocks are low, and others may not be convinced of the need for or effectiveness of IPT.

- Some TB-related data are not integrated with the electronic AMPATH medical records.

- Due to the recent stock-out of isoniazid, a valid historical baseline is not available. A study baseline will be collected in late September 2013.

Given the challenges for the clinical reminders intervention and study listed above, our team will work with AMPATH on the following actions:

1. The TB Tech team will work with AMPATH to track changes in the demand for isoniazid on a site-bysite basis and take steps to ensure that a 3-month supply is on hand.

2. The TB Tech team will support AMPATH, in any way that they deem warranted, on their anticipated TB education campaign for health workers. According to AMPATH's plans, this campaign will focus on reinforcing AMPATH's standards for TB screening, referral of symptomatic patients for testing, IPT for symptom-negative and test-negative patients, use of patient encounter forms including specific TB data fields, and use of clinical reminder sheets including acting on chest X-ray reminders. 
3. The TB Tech team will work with AMPATH leaders in radiology, laboratory, and pharmacy to investigate ways to improve the integration of TB-related data into the AMRS.

4. The TB Tech team will triangulate AMRS TB-related data and pharmacy records of isoniazid pickup, stocking, and ordering to establish a valid and reliable pre-pilot baseline.

5. The TB Tech team will work with AMPATH to monitor availability of X-ray equipment and commodities (e.g., film) at all study sites (intervention and control) and patient referrals to sites with working X-ray.

6. The TB Tech team will develop TB clinical summary sheet screening reminders for all new patients (within the last 6 months) who have not been diagnosed with active TB, treated for active TB, or initiated IPT within the last 6 months.

7. The TB Tech team will develop varied TB reminders, working to provide consistent TB education messages and individually tailored messages, even though the underlying message is the same, to avoid provider habituation.

8. The TB Tech team will develop clinical summary sheet reminders for both initiating IPT and for confirmatory testing and initiation of treatment for patients with active TB. 
U.S. Agency for International Development I 300 Pennsylvania Avenue, NW

Washington, DC 20523

Tel: (202) 7I2-0000

Fax: (202) 216-3524

www.usaid.gov 